\title{
Yuanyang Hani terrace: World Heritage Area Based on tourism attraction. Study on landscape security pattern
}

\author{
Wang Siqi \\ College of Urban Construction, Yunnan Open University, Kunming, China
}

Keywords: landscape security pattern; tourism attraction; Hani terraced fields.

\begin{abstract}
Based on the analysis of tourism attraction in the study area, this paper uses GIS, RS and other technologies to select four scenic spots, including Niujiaozhai terrace, Bada terrace, Doyishu terrace and Laohuzui terrace, as the "source" of the terrace landscape and Hani cultural landscape. Build a resistance surface to build a landscape security pattern in the study area. It will provide reference for the planning of scenic spots and the attraction of tourism.

The term landscape ecological security pattern [1-4] was first put forward by Professor Yu Kong-jian of Peking University in 1995. The theory is based on the landscape ecology theory of Forman [5]. It is pointed out that the ecological security pattern refers to the spatial pattern formed by the factors that play a key role in ecological security and health, such as forest, landscape, wetland and so on. Unification or pattern. It consists of "source" area (core area), buffer area, ecological corridor (source-to-source connection), radiation channel and strategic point (ecological node). Based on the tourism attraction analysis of the study area, this paper chooses the appropriate resistance factors and uses the minimum cumulative resistance model to construct the resistance surface to construct the landscape security pattern of the study area.
\end{abstract}

\section{Research scope and general situation}

The study area is located in the southwest of the Hani and Yi Autonomous Prefecture of Honghe River. The whole area has complex topography and steep topography, all of which are mountainous areas with large slope and terraced fields distributed in patches. The altitude ranges from $144 \mathrm{~m}$ to 2939.6 M. It has a large difference, a distinct three-dimensional climate and abundant rainfall. It is a subtropical monsoon climate. It includes Xinjie Town, Niujiaozhai Township, Panzhihua Township and Shengcun Township. In order to study the heritage area more comprehensively, this paper selects a total of 54322 hectares of area including the heritage core area and buffer area as the research scope, covering the Hani terrace, Hani settlement, natural landscape and cultural landscape.

\section{Data sources}

The basic data in this paper are derived from the PLAIDES-1 satellite remote sensing image in 2016 and DEM elevation data with a resolution of $2 \mathrm{~m}$. The administrative division map of the study area, landscape pattern map of the declared heritage area and scope map of the declared heritage area are taken as reference. Landscape security pattern is realized by ArcGIS10.2 interpretation and spatial analysis.

\section{Determination of resistance factors based on tourism attractiveness.}

In previous studies [6-8], the resistance factors were elevation slope, land use type, landscape index, vegetation coverage, precipitation, distance to residential areas, etc. Tourism attraction is the attraction degree of tourism resources to tourists. In other conditions, the more abundant tourism resources, the greater the degree of stimulation to tourists. Based on the analysis of the attraction of tourism resources in the study area, combined with the actual situation of this study, this paper 
makes a comprehensive analysis of the study area, taking into account the feasibility and maneuverability. The slope and land use types were selected as resistance factors for evaluation.

\subsection{Slope factor classification}

The terrace is the result of the effective transformation of the mountain area by the Hani people's wisdom. It is built along the slope along the direction of the contour line and forms a stepped or wavy section. Therefore, the bigger the slope, the better the landscape effect of the terrace, and the greater the attraction for tourists. According to the characteristics of terraced fields and slope gradient, the slope factors can be divided into five levels:

S1:0 15 degrees, S2:16 25 degrees, S3:26 35 degrees, S4:35 50 degrees, S1: > 50 degrees.

The resistance levels are divided into: S1 > S2 > S3 > S4 > S5.

\subsection{Factor classification of land use types}

Hani culture and terraced fields are the two landscapes in the study area, so the richer the landscape types on the surface, the greater the attraction for tourists, and the smaller the resistance. Based on the interpretation results of remote sensing maps, the study area is analyzed. The land use types mainly include construction land, water terraces, forests, shrubs, roads and water bodies. The comprehensive analysis of scenic resources shows that the terrace scenery and Hani cultural landscape agglomeration area are more attractive to tourists, and the relative resistance is the smallest. According to the expert consultation, the resistance levels of different land use types in the study area were divided into: terrace $<$ Construction Land $<$ forest $<$ shrub $<$ Water $<$ road $<$ unused land.

\section{Building a landscape safety pattern}

\section{1 determine "source"}

The study area includes Bada terrace, Doyishu terrace, Laohuzui terrace and Niujiaozhai Town terrace. Among them, Bada terrace, Doyishu terrace and Laohuzui terrace are relatively large in area. They contain natural elements of mountain area, terrace cultural landscape, material and cultural heritage of ancient villages and intangible terrace-ancient settlements. Cultural heritage, rice culture and Hani culture are characterized by biodiversity, landscape heterogeneity and human-landscape symbiosis. The four-dimensional isomorphism of water system-forest-village-terraced fields makes the region a highly coordinated, sustainable and benign ecological system. . Except for Niu Jiao Zhai terraced fields, other scenic spots are located in the core area of the world heritage.

The study area belongs to the dual heritage of the world cultural landscape, so the identification of the "source" in this area needs to consider Hani culture and terrace landscape. According to the comprehensive analysis, the area of Bada terrace, Doyishu terrace, Huzui terrace and Niujiaozhai

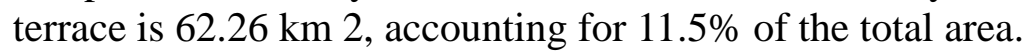

\subsection{Establish resistance surface}

The minimum cumulative resistance model (MCR) was established, and ArcGIS 10.2 was used to calculate the resistance surface to overcome the resistance to reach the "source". The minimum cumulative resistance model proposed by Knaapen[9-10] et al. Is as follows:

$$
M C R=f \min \sum_{j=n}^{i=m}\left(D_{i j} \times R_{i}\right)
$$

Where $\mathrm{f}$ is an unknown positive function, it reflects the distance relation from any point in the space to all sources. Dij is the space distance from the source J (cultural landscape) to a certain landscape unit plane $\mathrm{i}$, and $\mathrm{Ri}$ is the resistance value of landscape unit plane $\mathrm{I}$ to the movement of tourists. The resistance surface reflects the potential possibility and trend of species movement. In this study, the range and boundary of the resistance surface at different safety levels are determined 
by determining the mutation of the minimum cumulative resistance contour. According to the distribution of the comprehensive resistance value, the resistance value is divided into three grades.

The resistance surface around the source is divided into three grades, and the resistance value increases progressively. Among them, the first layer of expansion from the "source" is the low resistance horizontal plane with a pattern area of $134.63 \mathrm{~km} \mathrm{2,} \mathrm{which} \mathrm{is} \mathrm{the} \mathrm{buffer} \mathrm{zone} \mathrm{of} \mathrm{the} \mathrm{dual}$ heritage area of cultural landscape. Traditional villages with ethnic characteristics and a few terraces are also distributed in the buffer zone, and the second layer is the region with strong tourism attraction besides the "source" which needs to be protected. In the middle resistance level, the

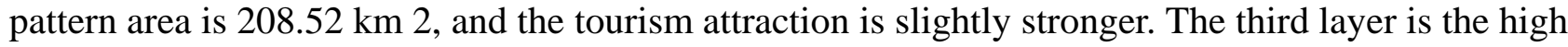

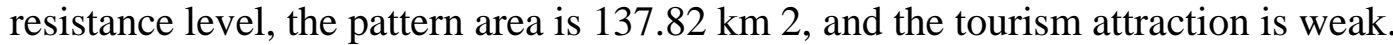

\section{Identification of ecological corridors, radiation channels and ecological nodes}

\subsection{Corridor recognition}

Corridor is a low cumulative resistance valley line connecting "source" and "source" in the study area. It is often some linear landscape elements such as water system, valley, mountain road, etc. It connects the natural and cultural landscape of the study area, plays an important role in constructing the network system of the entire heritage area, and ensures the integrity and continuity of the landscape. This paper uses the spatial analysis function of ArcGIS 10.2 to identify the potential habitat corridors and calculate the lowest resistance channel between adjacent "sources".

\subsection{Radiometric recognition}

Radiation tract is a low-lying force line around the "source" in the study area. It is an effective way for landscape elements to spread outward based on the "source", like a dendritic distribution.

\subsection{Strategic point recognition}

The tangent point of the iso-resistance value line between the adjacent "source" is the strategic point [1]. It connects four key nodes of the natural and cultural landscape core area and is the springboard for the direct transition of the scenic area. The determination of the strategic point can improve the tightness of the connection between the scenic areas and has important strategic significance for improving the attractiveness of the landscape.

\section{Construction of landscape safety pattern}

The spatial overlay function of ArcGIS 10.2 is used to overlay the distribution maps of "source" areas, corridors, radiation channels and strategic points identified above, thus forming the landscape security pattern of the study area.

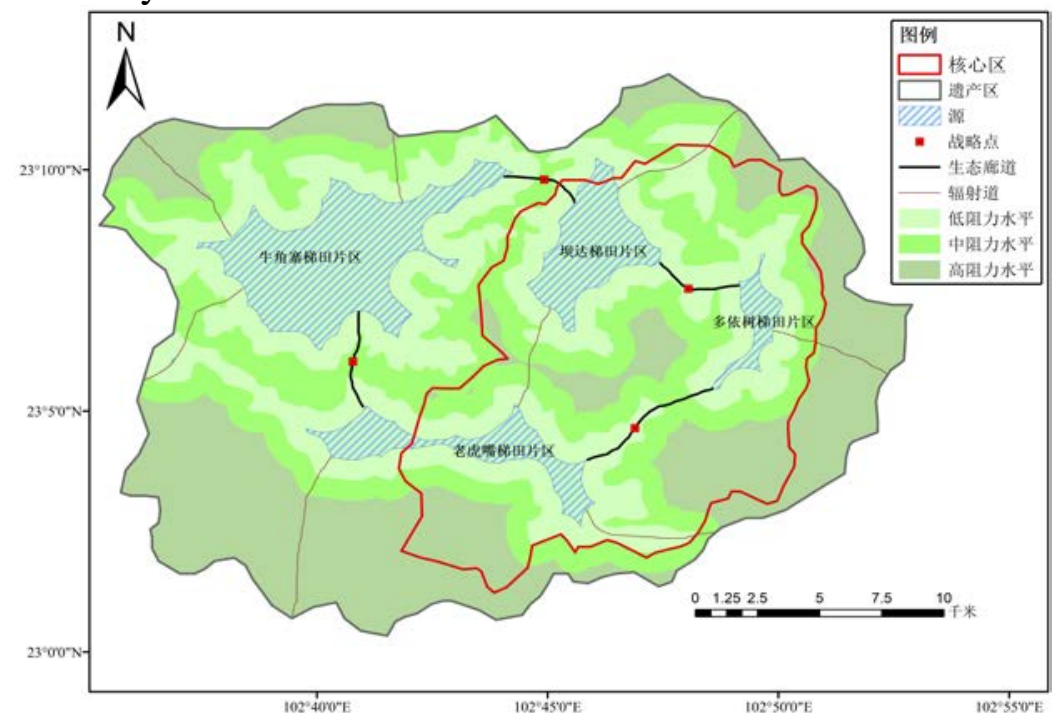

Fig. 1 Landscape security patterns in research area 


\section{Conclusion and discussion}

Bada terrace, Doyishu terrace, Laohuzui terrace and Niujiaozhai terrace are the four "source" areas, which are rich in tourism resources and have the greatest attraction.

Around the source, the low resistance level area, the middle resistance level area and the high resistance level area were divided, accounting for $24.78 \%, 38.36 \%$ and $25.37 \%$ of the land respectively. Among them, the natural and cultural landscape in the "source" area is more concentrated, and the ecology is fragile, so the key protection is needed to ensure the attraction of tourists. The low drag level zone is the nearest buffer from the source, which needs to be focused in the future development.

The selection of resistance factors still needs to be improved. It is the first attempt to select slope and land use type as resistance factors for tourism attraction. Other factors affecting the attractiveness of tourism can be taken into account, or cultural and natural landscape related factors can be extracted separately for the dual landscape heritage areas, and ecological security pattern can be constructed and superimposed. These are all need to be further explored.

\section{Acknowledgement}

Supported by the research fund of Yunnan Provincial Department of Education (2017ZDX224)

\section{References}

[1] Yu Kongjian. Landscape ecological security pattern of biological protection [J]. Acta ecology, 1999,19 (1): 8-15..

[2] Yu K - J. Security Patterns in Landscape Planning: W itha Case in South China. Doctoral Thesis, Harvard University, 1995.

[3] Yu K - J. Security patterns: a defensive approach toward landscape and environmental planning. In, Sellis T and GeorgoulisD (eds.), Proceedings, Athens International Conference, Urban Regional Environmental Planning and Informatics to Planning in An Era of Transition. National TechnicalU2n iversity of Athens, Faculty of Architecture Dept. of Urban and Regional Planning. 1997, 453 - 463.

[4] Yu Kong-jian, Li Di-hua, Liu Hailong. [M] "Counter-planning" Approach. China Construction Industry Press, 2005, 9:29-30.

[5] Forman R T T . Some general principles of landscape and regional ecology. Landscape Ecology, 1995, 10 (3) : 133 - 142.

[6] Yu Kong-jian, Qiao Qing, Li Di-hua, et al. Study on ecological land use based on landscape security pattern analysis: a case study of Dongsan Township, Beijing [J].Journal of Applied Ecology, 2009, 20 (8): 1932-1939.

[7] Guo Ming, Xiao Binning, Li Xin. Landscape Ecological Security Pattern Analysis of Jiuquan Oasis in Heihe River Basin [J].Acta Ecologica Sinica, 2006,26(2): 457-466.

[8] Li Hui, Yi Na, Yao Wengong, Wang Siqi, Li Zhiying and Yang Shuhua. Ecological land use planning in Shangri-La County based on landscape security pattern [J].Acta Ecologica Sinica, 2011, 31 (20): 5928-5936.

[9] Wang Yao, Gong Huili. Landscape Accessibility Analysis Based on Minimum Cumulative Resistance Model [J].Geospatial Information, 2007,5(4):45-47. [10]Knaapen J P,Scheffer M, Harms B. Estimating habitat isolation in landscape planning[J].Landscape \& Urban Planning.1992,23 (1):1-16.

[10] Yu K. Ecological Security patterns in landscapes and GIS application [J]. Annals of GIS, 1995, 1(2):88-102. 